\title{
त्री \\ O manuscrito de Einstein de 1912 como pista para o desenvolvimento da teoria da relatividade restrita
}

John STAGHEL

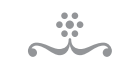

RESUMO

Examina-se o manuscrito escrito por Einstein em 1912, mas não publicado, que faz uma revisão da teoria da relatividade restrita. Nele, identificam-se algumas pistas sobre como Einstein desenvolveu suas idéias antes de 1905. Em particular, a explicação dada para o experimento de Fizeau indica como Einstein pôde manter o princípio de constância da velocidade da luz, mesmo parecendo ser incompatível com o princípio de relatividade.

Palavras-chave • Einstein. Relatividade restrita. Experimento de Fizeau. Constância da velocidade da luz.

\section{INTRODUÇÃO}

Em 1912, pediu-se a Albert Einstein que escrevesse um artigo de revisão sobre a teoria da relatividade (naquela época não havia ainda uma teoria da relatividade geral, de forma que não era necessário qualificar a teoria como "restrita"). Trata-se do artigo de revisão mais detalhado que Einstein escreveu, mas ele não chegou a ser publicado durante sua vida, ${ }^{\mathbf{1}}$ por motivos apresentados em detalhe na introdução editorial desse manuscrito (ver Einstein 1995 [1912], p. 3-8).

As seções 5,6 e 7 do manuscrito apresentam um argumento detalhado para a adoção dos dois princípios da teoria da relatividade restrita, argumento este que parece estar intimamente ligado ao caminho histórico que Einstein de fato trilhou para sua

1 Este texto foi publicado nas obras completas de Einstein, ver Einstein (1995 [1912]). A paginação que apresento nas referências em meu texto refere-se às páginas introduzidas pelos editores das obras completas. Incluí também as notas editoriais, que estão nas p. 102-8, pois os editores colocaram vários trechos do manuscrito nessas notas. Há também uma edição em fac-símile, Einstein (1996 [1912]), que apresenta o fac-símile nas páginas ímpares e a tradução para o inglês nas páginas pares. Todas as traduções do alemão para o inglês, no presente artigo, são minhas. 
adoção. Há vários razões para esta afirmação, algumas internas ao próprio documento, outras baseadas na semelhança entre os argumentos nele presentes e outros relatos que Einstein deu, na época e posteriormente, sobre o seu caminho para a teoria da relatividade restrita. Aqui me concentrarei nas razões internas, fazendo referência apenas ocasional a outros elementos de evidência. Em particular, mostrarei que Einstein fornece aqui a explicação mais completa do papel de experimento de Fizeau ${ }^{2}$ em seus argumentos anteriores a 1905, explicação esta que lança muita luz sobre os comentários mais enigmáticos sobre este assunto que ele fez alhures, tornando mais claro do que nunca o papel crucial que o experimento teve no desenvolvimento de seu pensamento anterior a 1905 sobre a óptica de corpos em movimento. Em particular, ele explica como o experimento o levou a abandonar toda resistência ao princípio da constância da velocidade da luz (ou, de forma abreviada, "princípio da luz"), apesar do fato de que ele queria manter o princípio de relatividade, com o qual o princípio da luz parecia ser incompatível.

Primeiro, algumas palavras sobre o estado do manuscrito. As seções 6 e 7 foram extensamente retrabalhadas, contendo numerosas passagens que estão riscadas (para eliminá-las) nas p. 18, 19, 20 e 21, e suplementadas por uma folha adicional, designada "p. 20a" pelos editores, escrita com uma tinta diferente. As passagens riscadas contêm um relato de possíveis alternativas para o princípio da luz, e de argumentos contra a adoção dessas alternativas, sem fazer menção a estrelas duplas. Já a p. 2oa contém um relato do argumento de Willem de Sitter contra as teorias de emissão, que se baseia nas observações de estrelas duplas. Faço a conjectura de que esta página teria sido adicionada posteriormente às outras páginas, depois que Einstein ficou ciente do argumento de de Sitter, ${ }^{3}$ que ele considerou definitivo. ${ }^{4}$ Conjeturo também que as passagens originais riscadas descreveriam seu enfrentamento anterior com uma teoria da emissão

$2 \mathrm{O}$ experimento em questão foi realizado por Armand Hippolyte Fizeau em 1851, e consistiu da transmissão de luz em meio a um tubo com água em movimento. O feixe de luz era dividido em duas partes, uma que rumava no mesmo sentido que a água, e outra que rumava contra. Usando técnicas pioneiras de interferometria, Fizeau detectou um deslocamento muito pequeno das franjas em relação ao caso em que a água estava em repouso. Isso foi interpretado como indicando que o éter seria parcialmente arrastado com a água, em acordo com uma fórmula derivada anteriormente por Augustin Fresnel, e em discordância com a hipótese de George Stokes de arrastamento total do éter pela matéria em movimento. (N.T.)

30 trabalho do astrônomo holandês foi publicado em 1913, ver Sitter (1913). No entanto, a maneira grotescamente errônea pela qual Einstein grafou o nome de de Sitter no manuscrito, "Pexider", sugere que ele ficou sabendo do trabalho oralmente antes de sua publicação. Uma carta de Einstein a Paul Ehrenfest de 28 de maio de 1913 (ver Klein et al., 1993, p. 523) sugere que foi Ehrenfest quem informou Einstein do trabalho de de Sitter.

4. "Die Unhaltbarkeit dieser Auffassung kann wohl als endgültig erwiesen betrachet werden" (Einstein, 1996 [1912], p. 35 ). ("A indefensabilidade desta abordagem pode de fato ser considerada como finalmente demonstrada”). 
da luz, assim como com uma outra alternativa possível ao princípio da luz que até hoje não foi discutida na literatura, e descreveriam as razões para seu abandono dessas tentativas de evitar o princípio da luz. Retornemos agora aos argumentos das seções 5 , 6 e 7 .

\section{Seção 5, intitulada “Princípio da Gonstância da Velocidade da luz"}

Em sua palestra de Kyoto em 1922, relata-se que Einstein teria dito:

Foi há aproximadamente 17 anos que veio a mim a idéia de tentar desenvolver o princípio de relatividade. Está claro que não posso dizer de maneira definitiva de onde esta idéia veio. Estou certo, porém, que ela originou do problema da óptica de corpos em movimento. 5

E de fato Einstein inicia a seção 5 de seu manuscrito de 1912 com uma discussão do princípio da constância da velocidade da luz. Primeiro ele desenvolve o princípio como uma conseqüência da teoria eletrodinâmica de Lorentz.

Einstein havia considerado a versão de Lorentz da teoria de Maxwell nas seções anteriores de seu manuscrito, e nesta seção ele mostra como as equações de Maxwell que ele denota coletivamente como "(I)" -, consideradas no vácuo, levam à equação da onda para cada componente dos campos elétrico e magnético, sendo $c$ a velocidade de propagação. Note-se que seu relato da teoria de Lorentz até este ponto no manuscrito não vai além da teoria conforme apresentada pelo físico holandês em seu Versuch de 1895 (Lorentz, 1895), o único trabalho de Lorentz com o qual Einstein tinha familiaridade em 1905 , segundo o que posteriormente escreveu. ${ }^{6}$

Ele conclui assim esta breve seção:

Podemos então, em harmonia com a teoria de H. A. Lorentz, montar o seguinte teorema fundamental [Grundsatz], que chamamos "o princípio de constância da velocidade da luz":

"Há um sistema de coordenadas em relação ao qual todo raio de luz no vácuo propaga com a velocidade $c . "$

5 Citação retirada de Stachel (2002 [1982]), p. 180. A fonte original em japonês está mencionada na nota 4 desta referência, p. 186.

6 Ver carta de Einstein para Carl Seelig, de 19 de fevereiro de 1955. 
Este teorema inclui uma asserção de alcance geral. Ela afirma que a velocidade de propagação da luz não depende nem do estado de movimento da fonte nem do estado de movimento dos corpos que circundam a região de propagação. A questão de até que ponto este teorema pode ser considerado verdadeiro é de fundamental significado para a teoria da relatividade. Por hora, nos contentaremos com o insight [Einsicht] de que ele é exigido pela teoria de Lorentz (Einstein, 1995 [1912], p. 16).

Aqueles que estão familiarizados com outros relatos de Einstein sobre o princípio da luz, e com discussões históricas posteriores, notarão uma peculiaridade a respeito do presente relato. A primeira asserção, de que a velocidade da luz é independente do movimento de sua fonte, é familiar. A segunda, de que ela é independente do estado de movimento dos corpos circundantes à região de propagação, não é. Veremos o significado desta segunda asserção na Seção 7, mas por enquanto noto apenas que ela é peculiar ao relato de Einstein no manuscrito de 1912.

\section{Seção 6, intitulada "O PRincípio de Relatividade”}

Esta seção está dividida em duas subseções. Na primeira, intitulada "O princípio de relatividade na mecânica clássica”, Einstein considerou um sistema mecânico fechado e mostrou que se houver um referencial $K$ no qual o sistema segue as leis de Newton, então o sistema seguirá essas leis em qualquer referencial $K$ ' que se move a uma velocidade constante com relação ao primeiro. Usou "as equações de transformação da cinemática costumeira" para relacionar as coordenadas espaciais e o tempo dos dois referenciais, ou seja, as seguintes equações para um movimento que se dá na direção $x$ - notando-se que Einstein as denota coletivamente como "(II)":

$$
\begin{aligned}
& x^{\prime}=x-v t \\
& y^{\prime}=y \\
& z^{\prime}=z \\
& t^{\prime}=t
\end{aligned}
$$

Chamando qualquer referencial no qual as equações de Newton são válidas de "um sistema "legítimo" ["ein 'berechtigtes' System”], ele enuncia "o princípio de relatividade da mecânica clássica":

Todo referencial que está em movimento de translação uniforme com respeito a um referencial legítimo é, por sua vez, um referencial legítimo. Com relação a todos esses sistemas legítimos, as leis do movimento de qualquer sistema (mecânico) são as mesmas (Einstein, 1995 [1912], p. 17). 
Talvez valha a pena notar que aqui, assim como no artigo de 1905, Einstein não utiliza o termo "sistema inercial", que Ludwig Lange havia introduzido nos anos 1880 para designar o que Einstein chama aqui de "sistema legítimo".

A segunda subseção é intitulada "Princípio de relatividade e experiência". Ela diz respeito ao fracasso de todas as tentativas de encontrar quaisquer efeitos físicos locais devidos ao movimento translacional da Terra com relação a um referencial "legítimo" ["brechtigte"] definido na astronomia, com origem fixada no centro de massa do sistema solar e cujos eixos apontam em direções de pontos definidos nos céus, conforme estabelecidos pelas estrelas fixas. Einstein não menciona, mas este é justamente o referencial escolhido por Newton no Livro III do Principia para representar o estado de repouso absoluto. ${ }^{7}$ Do resultado negativo de todas essas tentativas, tanto mecânicas quanto não-mecânicas, ele conclui:

Portanto, o princípio de relatividade enunciado anteriormente torna-se um fato quase indubitável [unzweifelbarer Tatsache], e de fato ele parece ser válido não só na mecânica, mas também em todos os campos da física (Einstein, 1995 [1912], p. 18).

Curiosamente, apesar de ele mencionar que há muitos desses experimentos, ele cita apenas o experimento de Michelson-Morley, que ele diz ser "fundamentalmente importante". Ele não menciona o conhecido experimento de pensamento do condutor magnético, com o qual abre o artigo de 1905, como evidência a favor do princípio de relatividade (Einstein, $195^{8}$ [1905]). Mas a razão disso pode bem ser que sua discussão está confinada à busca por efeitos do movimento translacional da Terra e a experimentos de fato e não de pensamento.

Ele conclui a seção com o seguinte comentário:

Acredito, portanto, que a validade do princípio de relatividade mal pode mais ser posta em dúvida (Einstein, 1995 [1912], p. 18).

Conforme discuti em outras ocasiões, ${ }^{8}$ muito antes de o manuscrito de 1912 estar disponível, toda a evidência histórica apontava para o fato de que Einstein ficara convencido da validade do princípio de relatividade muito antes de ter completado a teoria da relatividade.

7 Ver o livro iı de Newton (1999 [1687]), em especial "Hipótese i: O centro do sistema do mundo está em repouso", p. 816. Talvez seja de relevância mais imediata o tratamento feito por Ernst Mach do conceito de espaço absoluto, em seu Mechanik (Mach, 1988 [1883]), tratamento este que Einstein leu várias vezes antes de 1905.

8 Ver Stachel (2002 [1982]), Stachel (2002 [1983]), Stachel (2002 [1989]). 


\section{SeçÃo 7, "Aparente incom PATIBILIDAdE do PRINGípio DA GONSTÂNGIA DA VELOGIDADE DA LUZ GOM O PRINGÍPIO DE RELATIVIDADE"}

Estamos chegando ao cerne da questão. Einstein começa notando que:

Dificilmente tantos experimentos teriam sido realizados para demonstrar uma influência do movimento da Terra em experimentos terrestres se a teoria de Lorentz, conforme foi apresentada, não parecesse incompatível com o princípio de relatividade (Einstein, 1995 [1912], p. 19).

Einstein continua para demonstrar isso com mais detalhe. Em um artigo de resenha de 1915 , ele tece comentários semelhantes a respeito desse assunto:

A teoria de Lorentz agora desperta nossa desconfiança porque ela parece contradizer o princípio de relatividade. [...] Os sucessos da teoria de Lorentz foram tão significativos que os físicos teriam abandonado, sem hesitar, o princípio de relatividade, se um resultado experimental importante não existisse, do qual devemos agora falar, que é o experimento de Michelson. ${ }^{9}$

Voltando ao texto de 1912, aparece agora o primeiro trecho que seria posteriormente riscado:

Esta situação nos compele novamente a duvidar da correção do princípio da constância da velocidade da luz e, junto com ele, as equações fundamentais (I) da teoria de Lorentz. Devemos pesar a possibilidade de que, apesar dos sucessos da teoria de Lorentz, talvez o princípio da constância da velocidade da luz não seja estritamente válido; mas devemos reter o princípio de relatividade e também as equações cinemáticas II, isto é, a lei do paralelogramo de forças.

Se nos colocarmos neste ponto de vista, o experimento de Fizeau nos obriga a concluir (afora a dispersão) que várias velocidades da luz devem ser possíveis em um meio transparente. Se de fato a velocidade da luz em um meio fosse sempre $V_{\circ}{ }^{10}{ }^{10}$ conforme o julgamento de um observador que se move com o meio, então,

9 Referência e versão em alemão encontram-se em Stachel (2002 [1982], p. 180).

10 Note-se aqui como Einstein, assim como no restante desta seção, abandona sua notação $c$ para a velocidade da luz, que ele adotara anteriormente no manuscrito, e passa a denotá-la por $V$, que é a notação que ele usou no seu artigo de 1905 . Esta era presumivelmente a notação que ele usou em suas notas de pesquisa anteriores e não preservadas, sugerindo novamente que as considerações desta seção refletiriam seus argumentos internos anteriores. 
de acordo com a lei de paralelogramo de velocidades, a velocidade da luz para um observador que não está se movendo com o meio seria $V_{\circ}+q l$, o que está em contradição com o resultado experimental. Somos então obrigados a assumir que, no experimento de Fizeau, a velocidade da luz relativa ao meio em movimento é diferente daquela no mesmo meio quando em repouso. Dado que isso também deve valer se o meio for opticamente passivo [unwirksam], isto é, quando $n=1$, então (Einstein, 1995 [1912], p. 19).

Após este trecho riscado, interrompido no meio da frase, o texto continua:

Mais exatamente, vimos até agora apenas que as três coisas seguintes são incompatíveis

a) o princípio de relatividade

b) o princípio da constância da velocidade da luz (teoria de Lorentz)

c) as equações de transformação (II), ou equivalentemente a lei do paralelogramo de velocidades.

Chega-se à teoria conhecida como "teoria da relatividade" se forem mantidas a) e b), rejeitando-se c). A seguir mostraremos que este modo de proceder é possível. Mas qualquer um que não tenha se ocupado profundamente com a eletrodinâmica de corpos em movimento estará certamente inclinado a manter a) e c) e abandonar b). Assim, partindo deste último ponto de vista, tirarei algumas conseqüências, de forma a assim justificar o ponto de vista da teoria da relatividade (Einstein, 1995 [1912], p. 20).

A seguir, ele começa sua discussão detalhada do experimento de Fizeau, do ponto de vista que rejeita o princípio de constância da velocidade da luz.

Se olharmos para o arranjo do experimento de Fizeau, em princípio, precisamos distinguir os seguintes objetos

1) A fonte de luz $(L)$

2) O meio atravessado pela luz $(M)$

3) A Terra com todos seus objetos restantes $(E)$.

Podemos caracterizar de modo suficiente a situação no experimento de Fizeau da seguinte maneira: $L$ e $E$ estão em repouso com relação ao sistema de coordenadas $K$. $M$ é atravessado pela luz (velocidade da luz $V$ ) na direção do eixo $x$ e se move nesta direção com velocidade $q l$. De acordo com a), esta situação é equivalente à seguinte: $M$ está em repouso; $L$ e $E$ estão com velocidade - $q$ l ao longo do 
eixo $x$. A velocidade da luz $V$ ' relativa a $M$ é, de acordo com o resultado de Fizeau e c), igual a

$V^{\prime}=\left\{V_{\circ}+q l\left(1-1 / n^{2}\right)\right\}-q l=V_{\circ}-q l / n^{2}$,

onde $V_{\circ}$ é a velocidade da luz em $M$, no caso em que $L, M$ e $E$ estão em repouso mútuo entre si. Assim, de acordo com este ponto de vista, o fato de que $L$ e $E$ estão se movendo com velocidade - $q l$ em relação a $M$ altera a velocidade da luz em $M$ por um fator $q l / n^{2}$. Pode-se tomar tanto o movimento de $E$ quanto o de $L$ como responsável pela mudança $V^{\prime}-V_{\circ}$ (Einstein, 1995 [1912], p. 20).

O restante desta seção passa a levar em consideração cada um desses dois casos, para mostrar que cada qual leva a complicações mais problemáticas do que o abandono de c). Conforme notado no começo do presente artigo, a primeira tentativa é novamente riscada e substituída pela discussão do trabalho de de Sitter. Mas é ela que nos interessa aqui, pois poderia bem representar os argumentos internos que Einstein usou durante o período em que ele pensava que teria que abandonar o princípio da luz e, com ele, a teoria de Lorentz. Einstein altera sua numeração dos dois casos, de forma que, ao traduzir seus comentários, omitimos a numeração em favor da menção explícita de cada caso.

Referindo-se ao caso em que o movimento da Terra é tido como responsável pela alteração na velocidade da luz, ele escreve que isso

[...] leva a grandes complicações. Pois a influência do movimento de $E$, em relação a $M$, na velocidade da luz em $M$, deve ser menor, quanto menor for a massa (?) de $E$ e quanto maior for feita a massa de $M$. No caso do experimento de fato, a situação pode ser concebida da seguinte maneira: a massa efetiva de $E$ é tão grande, comparada com a de $M$, que só ela precisa ser levada em consideração. Porém, dado que não há nenhuma indicação de uma lei de acordo com a qual as massas em movimento poderiam modificar a velocidade da luz no espaço circundante, de acordo com este ponto de vista uma liberdade arbitrária estaria em jogo. De qualquer forma, tal ação-de-massa deve diminuir rapidamente com a distância, senão entrar-se-ia em conflito com o resultado do experimento de Michelson (Einstein, 1995 [1912], p. 20).

No caso em que o movimento da fonte de luz é tido como responsável:

[...] a velocidade da luz em $M$ dependeria da velocidade da fonte de luz relativa a $M$ (Ritz e Ehrenfest) (Einstein, 1995 [1912], p. 20). 
Aqui ele está evidentemente se referindo às tentativas de desenvolver uma teoria balística da luz, uma idéia que ele alhures reconheceu que teve que enfrentar antes de 1905, e que mais recentemente foi discutida por Walther Ritz e Paul Ehrenfest. De fato, no volume 5 de suas Obras completas (cf. Klein et al., 1993) há uma troca de cartas entre Einstein e Ehrenfest justamente sobre esse tópico. E pode bem ser que tenha sido o interesse de Ehrenfest em discutir tal teoria balística que levou Einstein a discutir a questão tão amplamente em seu artigo de revisão. Continuando a citação de seu manuscrito de 1912:

Então em $M$ poderia haver raios de luz com todas as velocidades de propagação possíveis, arbitrariamente pequenas e arbitrariamente grandes. Uma onda plana não seria definida somente por sua intensidade, cor e estado de polarização; terse-ia que somar a velocidade como um elemento adicional de informação para determiná-la, o que porém não poderia ser detectado junto a todos os efeitos de primeira ordem (que seriam proporcionais à primeira potência da velocidade da fonte de luz). Pois a luz, quando provém de estrelas que se movem em relação à Terra, tem - até onde vão nossos experimentos - as mesmas propriedades que a luz de fontes terrestres. Para estar de acordo com este ponto de vista, é preciso assumir as suposições mais estranhas; por exemplo, a seguinte: se a luz de velocidade $c+v$ incide perpendicularmente em um espelho, então a luz refletida teria velocidade $c-v$. Essas complicações fazem parecer compreensível que, até agora, ninguém tenha conseguido montar equações diferenciais e condições de contorno que estivessem de acordo com este ponto de vista (Einstein, 1995 [1912], p. 20).

Podemos agora entender o significado das duas cláusulas na formulação de Einstein do princípio da constância da velocidade da luz: "a velocidade de propagação da luz não depende nem do estado de movimento da fonte nem do estado de movimento dos corpos que circundam a região de propagação". Ele tem em mente o experimento de Fizeau e as duas explicações alternativas possíveis do seu resultado, no caso de se insistir em manter a lei galileana da adição de velocidades (que ele chama de "lei do paralelogramo das velocidades"). Nenhuma é compatível com sua formulação do princípio da luz; e, inversamente, seus argumentos contra cada uma das duas alternativas tornam claro porque ele decidiu manter o princípio da luz (o princípio de relatividade não estava em questão a essa altura no desenvolvimento de seu pensamento) e abandonar a lei galileana de adição da velocidades.

Na p. 2oa, que foi evidentemente adicionada posteriormente, Einstein apresenta o trabalho de de Sitter como argumento decisivo contra a tese de que o movimento de $L$ seja a causa da mudança da velocidade da luz em $M$ - um argumento ao qual ele clara- 
mente não podia recorrer quando estava tentando resolver esse problema antes de 1905 . No entanto, ele faz alguns comentários sobre a outra possibilidade que poderia ser relevante para seu pensamento pré-1905. Nesse caso,

[...] o movimento da matéria circundante teria uma influência na velocidade de propagação da luz no meio em repouso. A influência do movimento da matéria, através do qual a luz passa, no resultado do experimento de Fizeau poderia então ser associada ao fato de que a Terra está em movimento em relação ao meio irradiado. Ter-se-ia que supor que o resultado do experimento de Fizeau seria diferente se ele fosse conduzido em um corpo celeste suficientemente pequeno. Mesmo que esta abordagem não possa ser considerada definitivamente refutada, sua correção já é improvável devido à sua extraordinária complicação. É difícil acreditar que a velocidade da luz em um meio dependa do estado de movimento dos corpos circundantes, mas isso só até certo ponto, quando se considera que esses corpos podem possuir uma massa muito grande em comparação com a do corpo irradiado. Até agora ninguém conseguiu fundar uma teoria útil sobre esta suposição (Einstein, 1995 [1912], p. 20a).

Essa idéia é deveras curiosa, e até onde eu sei não se encontra na literatura contemporânea. Assim, é difícil evitar a impressão de que Einstein aqui está nos fornecendo um vislumbre de seu pensamento anterior.

Tendo levado o leitor a abandonar qualquer oposição ao princípio da luz, Einstein agora passa para a demonstração, prometida anteriormente, de que o princípio de relatividade e o princípio da luz são de fato compatíveis e que a lei galileana de adição pode ser abandonada, se "nós investigarmos que tipo de significado as especificações [Angaben] espacial e temporal têm na física" (Einstein, 1995 [1912], p. 21). ${ }^{11}$ Mas aqui alcançamos um terreno que já nos é familiar, constando de suas outras publicações; assim, por mais interessante que seja sua exposição, finalizaremos este relato com alguns comentários adicionais.

\footnotetext{
11 Einstein atribuiu tal importância à questão da reconciliação do princípio de relatividade com o princípio da luz que, após mostrar que Mach apresentara em seu Mechanik as idéias que estão por trás do princípio de relatividade, Einstein teceu o seguinte comentário: "Não é improvável que Mach pudesse ter chegado ao princípio de relatividade se, à época em que sua mente ainda tinha o frescor da juventude [er jungendfrischen Geistes war], o problema do significado da constância da velocidade da luz já tivesse ocupado os físicos. Na ausência deste estímulo, surgido da eletrodinâmica de Maxwell-Lorentz, mesmo os clamores críticos de Mach não foram suficientes para despertá-lo para a necessidade de uma definição da simultaneidade de eventos distantes" (Einstein, 1996 [1916]).
} 


\section{Conclusão}

Anos mais tarde, Einstein mencionaria um "experimento de pensamento infantil" que lhe ocorrera com dezesseis anos, e que seria a semente de sua teoria da relatividade restrita. Reproduzo aqui o relato de Alexander Moszkowski em seu livro de 1921, que parece ser a versão mais antiga preservada e que não é tão bem conhecida quanto outras:

É um fato que já com o aluno de Aarau surgiram problemas que então se encontravam na periferia da pesquisa possível da época. Certamente ele não era nenhum cientista, mas o que ele com dezesseis anos procurava já penetrava em âmbitos de sua pesquisa ulterior. Trata-se aqui de simplesmente registrar o fato e renunciar a analisar o processo de gestação; afinal, porque deveríamos tentar elucidar o salto de pensamento que levou o jovem aluno de uma escola cantonal a entender com muita penetração uma física ainda quase totalmente fechada? O problema que ele estava considerando era o da óptica dos corpos em movimento, mais precisamente, o da luz emitida por corpos em movimento relativo ao éter. Aí se encontram os primórdios do grande complexo de idéias que tanto transformaria nossa visão do mundo. Não é falsa então a afirmação de um biógrafo que se arrisca a escrever que o início do percurso da teoria da relatividade remonta a essa época. ${ }^{12}$

Aproximadamente dez anos depois, Einstein escreveu para um psicólogo, que lhe inquirira sobre a natureza de sua motivação, descrevendo três questões chave que haviam motivado sua "própria obra de vida". Na primeira questão, podemos reconhecer uma versão abstrata, generalizada, da estória do raio de luz (citado em Stachel, 2002, p. 5):

Como a representação de um raio de luz depende do estado de movimento do sistema de coordenadas em relação ao qual ele se refere?

Em suas Notas Autobiográficas, Einstein (1982, p. 55; [1949], p. 53]) conta a estória com maiores detalhes e adiciona:

Desde o início, tive a intuição clara de que, segundo o ponto de vista desse observador [que persegue o feixe de luz com velocidade $c$ ], tudo devia acontecer de acordo com as mesmas leis aplicáveis a um observador que estivesse em repouso em relação à Terra. 
De fato, não era possível avançar mais na resolução dessa questão antes que Einstein adotasse o princípio de relatividade.

Sabemos de outras fontes que o fenômeno da indução eletromagnética desempenhou um papel importante na adoção inicial de Einstein do princípio de relatividade num estágio bastante inicial de sua pesquisa sobre a eletrodinâmica de corpos em movimento. Porém, quando ele percebeu a aparente incompatibilidade do princípio de relatividade com o princípio da luz, parece que o foco de sua atenção se dirigiu para a óptica dos corpos em movimento, numa tentativa de resolver essa incompatibilidade; primeiro, investigando as conseqüências do abandono do princípio da luz; e então, depois que ele se convenceu que tais tentativas seriam infrutíferas, percebendo como superar essa aparente incompatibilidade por meio de uma análise crítica do conceito de simultaneidade à distância. Sugiro que apenas depois que ele resolveu esse problema e desenvolveu sua nova cinemática é que ele retornou aos problemas da eletrodinâmica de corpos em movimento stricto sensu. Essa seqüência então se refletiria na estrutura do artigo de 1905 , com a sua divisão numa primeira parte cinemática e numa segunda parte eletrodinâmica.

Parece claro que a explicação do experimento de Fizeau desempenhou um papel importante no caminho de Einstein para seu artigo de 1905. Se, enquanto considerava alternativas para o postulado da luz, ele investigou a possibilidade de teorias do eletromagnetismo diferentes da de Lorentz, tais teorias quase certamente teriam incluído equações de campo em meios diferentes do vácuo - como a eletrodinâmica de Hertz, que seguia a invariância de Galileu, e que sabemos que Einstein conhecia em $1899 .{ }^{\mathbf{1 3}}$.

Traduzido do original em inglês por Osvaldo Pessoa Júnior

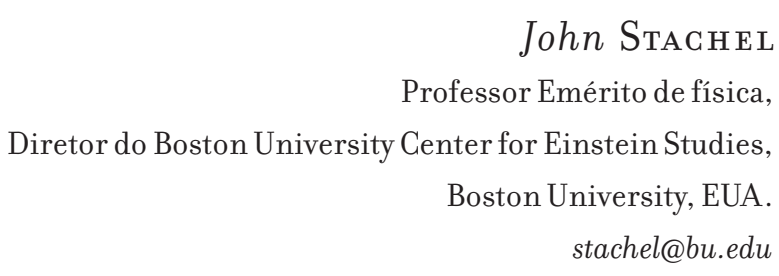

stachel@bu.edu

13 Ver carta de Einstein para Mileva Mari, de 10 de agosto de 1899 (Stachel, Cassidy \& Schulmann, 1987, p. 225-7). 


\begin{abstract}
One examines the unpublished manuscript written by Einstein in 1912, which reviews the theory of special relativity. Some clues are given in the manuscript for how Einstein developed his ideas before 1905 . In particular, the explanation given for Fizeau's experiment indicates how Einstein abandoned all resistance to the principle of the constancy of the speed of light, in spite of its apparent incompatibility with the relativity principle.
\end{abstract}

KEYwORDs • Einstein. Special relativity. Fizeau's experiment. Constancy of the speed of light.

\title{
REFERÊNCIAS BIBLIOGRÁFICAS
}

Einstein, A. Autobiographical notes. In: Schilpp, P. A. (Ed.). Albert Einstein:philosopher-scientist. La Salle: Open Court, 1949. p. 1-94. (Trad. em português: Notas autobiográficas. Trad. de A. S. Rodrigues. Rio de Janeiro: Nova Fronteira, 1982.)

. Sobre a electrodinâmica dos corpos em movimento. In: Lorentz, H. A.; Einstein, A. \& Minkowski,

H. Princípio de relatividade. Lisboa: Fundação Calouste Gulbenkian, $195^{8}$ [1905] p. 47-86.

Einstein's manuscript on the special theory of relativity. In: Klein, M.; Kox. A. J.; Renn, J. \& Schulmann, R. (Ed.). The collected papers of Albert Einstein. Princeton: Princeton University Press, 1995 [1912].v. 4: The Swiss years: writings 1912-1914. p. 9-108.

. Einstein's 1912 manuscript on the special theory of relativity: a facsimile. New York: George Braziller, 1996 [1912].

. Ernst Mach. In: Kox. A. J.; Klein, M. \& Schulmann, R. (Ed.). The collected papers of Albert Einstein.

Princeton: Princeton University Press, 1996 [1916]. v. 6: The Berlin years: writings 1914-1917. p. 278-81. (Original em: Physikalische Zeitschrift, 17, p. 101-4, 1916.)

Klein, M.; Kox. A. J.; \& Schulmann, R. (Ed.). The collected papers of Albert Einstein. Princeton: Princeton University Press, 1993. v. 5: The Swiss years: correspondence 1902-1914.

Klein, M.; Kox. A. J.; Renn, J. \& Schulmann, R. (Ed.). The collected papers of Albert Einstein. Princeton: Princeton University Press, 1995. v. 4: The Swiss years: writings 1912-1914.

Kox. A. J.; Klein, M. \& Schulmann, R. (Ed.). The collected papers of Albert Einstein. Princeton: Princeton University Press, 1996. v. 6: The Berlin years: writings 1914-1917.

LoRENTz, H. A. Versuch einer Theorie der elektrischen und optischen Erscheinungen in bewegten Körpern. Leiden: Brill, 1895 .

Lorentz, H. A.; Einstein, A. \& Minkowski, H. Princípio de relatividade. Lisboa: Fundação Calouste Gulbenkian, $195^{8}$.

МАGн, E. Die Mechanik in ihrer Entwicklung historisch kritisch dargestellt. Berlin: Akademie-Verlag, 1988 [1883].

Moszkowsкi, A. Einstein: Einblicke in seine Gedankenwelt. Hamburgo: Hoffmann \& Campe, 1921.

Newton, I. The principia. Mathematical principles of natural philosophy. Trad. de I. B. Cohen \& A. Whitman.

Berkeley: University of California Press, 1999 [1687].

Schilpp, P. A. (Ed.). Albert Einstein: philosopher-scientist. La Salle: Open Court, 1949.

Sitter, W. de. Ein astronomischer Beweis für die Konstanz der Lichtgeschwindigkeit. Physikalische Zeitschrift, 14, p. 479, 1913.

Stachel, J. Einstein from 'B' to 'Z'. Boston: Birkhäuser, 2002. 
"What song the syrens sang": how did Einstein discover special relativity?. In: Boston: Birkhäuser, 2002 [1983]. p. 157-69. Disponível em: <http://www.aip.org/history/einstein/essayeinstein-relativity.htm> Acesso em: 29 out. 2005.

Einstein and Michelson: the context of discovery and the context of justification. In:

Boston: Birkhäuser, 2002. p. 177-90. (Original em: Astronomische Nachrichten, 3०3, p. 47-553, 1982.)

Einstein on the theory of relativity. In: . Boston: Birkhäuser, 2002 [1989]. p. 191-214.

(Original em: Staghel, J.; Cassidy, D.; Renn, J. \& Schulmann, R. (Ed.). The collected papers of Albert Einstein. Princeton: Princeton University Press, 1989. v. 2: The Swiss years: writings 1900-1909. p. 253-74.)

Stachel, J.; Cassidy, D. \& Schulmann, R. (Ed.). The collected papers of Albert Einstein. Princeton: Princeton University Press, 1987. v. 1: The early years: 1879-1902.

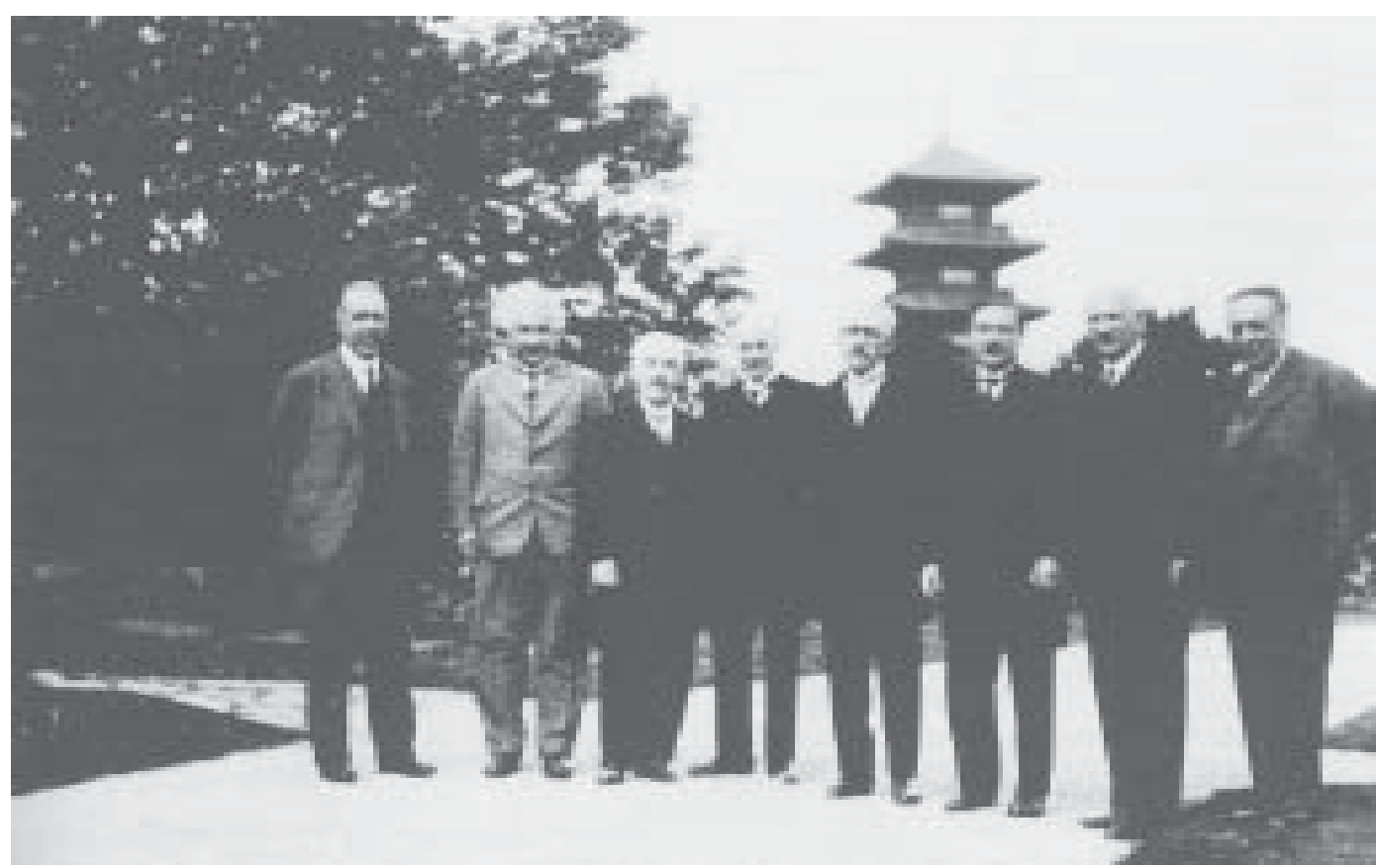

Einstein e outros membros da comissão de cientistas, reunidos na Bélgica em 1932, para planejar o Congresso Solvay de 1933. Niels Bohr está è esquerda de Einstein. 